Cross-sectional study

\section{Clinically significant pain is experienced by just over a third of all hospitalised patients, affecting around a half of surgical and a quarter of medical admissions}

10.1136/eb-2014-101937

\section{Elizabeth Manias}

Faculty of Health, Deakin University, School of Nursing and Midwifery, Burwood, The University of Melbourne, Melbourne School of Health Sciences, Royal Melbourne Hospital, Department of Medicine, Parkville, Victoria, Australia

Correspondence to: Professor Elizabeth Manias, Faculty of Health, Deakin University, School of Nursing and Midwifery, 221 Burwood Highway, Burwood, VIC 3125, Australia; emanias@deakin.edu.au

Commentary on: Carr EC, Meredith P, Chumbley G, et al. Pain: a quality of care issue during patients' admission to hospital. J Adv Nurs 2013;70:1391-404.

\section{Implications for practice and research}

- Greater attention should be placed on assessing pain independently of vital signs, when there is evidence of pain that needs to be relieved.

- Future research needs to examine the development and implementation of minimum standards for clinicians assessing and responding to pain in hospitalised patients.

\section{Context}

Despite advancements in pain control, patients' experience of pain continues to be a major problem. There have been no hospitalwide studies conducted on pain levels of whole populations of patients admitted to different clinical settings and monitored across time. This study involved examining patients' intensity of pain at different times during their inpatient stay. The authors explored clinically significant pain (CSP), which they defined as patients' pain scores that were in the severe or moderate range.

\section{Methods}

The sample comprised all documented pain scores for patient hospital admissions that occurred over a 1-year period. Data were collected at a district general hospital in the UK, using an electronic software system for routine documentation of vital signs and pain scores. Patients evaluated their pain intensity using a verbal rating scale with four categories: 0 (no pain); 1 (mild pain); 2 (moderate pain); and 3 (severe pain). Nurses entered data into the software system using handheld devices. They were also invited to enter additional pain intensity scores independently of vital signs. Descriptive statistics were reported, which involved evaluating trends of CSP in different clinical settings and during the patients' stay.

\section{Findings}

In total, 810774 pain scores were analysed, comprising 38451 patient stays. CSP occurred in $38.4 \%$ of patient stays. In considering the clinical settings, 51.3\% of all surgical admissions experienced CSP while $26.5 \%$ of all medical admissions experienced CSP. For 30.3\% of patients, initial CSP scores were followed sequentially by another score at the CSP level. For patients who were discharged alive, 5.4\% reported CSP, while for those who died, 7.5\% reported CSP in the final pain score. Intervals between pain scores were similar regardless of whether patients had CSP or not. In all, $0.2 \%$ of pain scores occurred independently of vital sign assessment.

\section{Commentary}

Through electronic devices at the bedside, real time data were accessed on pain scores to map the occurrence of CSP during patients' hospital stay. The study provided new knowledge about population trends about how pain scores varied in relation to the presence of consecutive pain scores, the interval between assessment of pain scores and the presence of pain at hospital discharge in terms of whether patients were alive or dead. The relatively large size of the pain database used in the study demonstrates major progression from previous work.

The results confirm that a gap exists between assessing pain and implementing effective strategies to manage pain. Of major concern, was the reoccurrence of CSP during patients' stay, despite the ability to mandatory screen for pain through electronic devices. Rather than focusing on educating clinicians to implement pain-relieving strategies, greater attention should be placed on changing the organisational culture in managing pain. Previous research has demonstrated complex barriers of the organisational culture that contribute to impeding improvements in pain care. ${ }^{1}$ In view of the serious consequences associated with poor pain management, policy directives need to be developed from understandings of these barriers to create major reform.

In this study, data were obtained from only one hospital, and therefore, the results may not necessarily reflect what occurs in other institutions. Pain was assessed using a four-point verbal rating scale, which may not be suitable for all patients. This scale may not be effective in older patients, or in patients who are unresponsive or who have cognitive or motor problems. ${ }^{2}$ Nevertheless, the use of a simple pain tool would have facilitated nurses' compliance with completing the tool. The investigators did not examine the pain management strategies that clinicians may have implemented to treat pain. It is possible that some patients could have experienced intractable or chronic pain, which was extremely difficult to resolve. More likely, it is probable that pain relief was inadequate, inappropriate or delayed.

The introduction of electronic support devices may encourage nurses to document pain scores, but by themselves, these devices are insufficient to facilitate improvements in pain practice. Linking pain intensity scoring with vital signs assessment may not be the most effective way to monitor pain, especially if vital signs are stable. Patient safety organisations need to work closely with hospital managers and clinicians to establish quality targets for improving patients' pain experience.

Competing interests None.
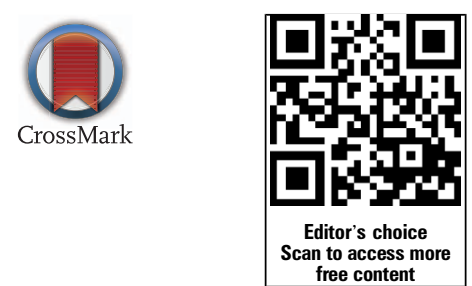

\section{References}

1. Manias E. Complexities of pain assessment and management in hospitalised older people: a qualitative observation and interview study. Int J Nurs Stud 2012;49:1243-54.

2. Clark W, Yang J, Tsui SL, et al. Unidimensional pain rating scales: a multidimensional affect and pain survey (MAPS) analysis of what they really measure. Pain 2002;98:241-7. 